\title{
Organic photovoltaics for simultaneous energy harvesting and high-speed MIMO optical wireless communications
}

\author{
Iman Tavakkolnia', Lethy K. Jagadamma², Rui Bian ${ }^{3}$, Pavlos P. Manousiadis², Stefan Videv', Graham A. Turnbull $\mathbb{O}^{2}$, \\ Ifor D. W. Samuel $\mathbb{B}^{2}$ and Harald Haas (1)
}

\begin{abstract}
We show that organic photovoltaics (OPVs) are suitable for high-speed optical wireless data receivers that can also harvest power. In addition, these OPVs are of particular interest for indoor applications, as their bandgap is larger than that of silicon, leading to better matching to the spectrum of artificial light. By selecting a suitable combination of a narrow bandgap donor polymer and a nonfullerene acceptor, stable OPVs are fabricated with a power conversion efficiency of $8.8 \%$ under 1 Sun and $14 \%$ under indoor lighting conditions. In an optical wireless communication experiment, a data rate of $363 \mathrm{Mb} / \mathrm{s}$ and a simultaneous harvested power of $10.9 \mathrm{~mW}$ are achieved in a 4-by-4 multipleinput multiple-output (MIMO) setup that consists of four laser diodes, each transmitting $56 \mathrm{~mW}$ optical power and four OPV cells on a single panel as receivers at a distance of $40 \mathrm{~cm}$. This result is the highest reported data rate using OPVs as data receivers and energy harvesters. This finding may be relevant to future mobile communication applications because it enables enhanced wireless data communication performance while prolonging the battery life in a mobile device.
\end{abstract}

\section{Introduction}

Wireless data access is a necessity in today's data-driven world, which affects all facets of modern societies, including health, commerce, politics, and education. The demand for mobile data has been growing at a rate of $60 \%$ annually over the past 13 years ${ }^{1}$, and there is no evidence to suggest that this exponential growth rate will slow down in the foreseeable future. In contrast, emerging new technologies, such as ultrahigh-definition video streaming, online virtual reality, online gaming, cloud-based services and the Internet of Things (IoT), require

Correspondence: Graham A. Turnbull (gat@st-andrews.ac.uk) or Ifor D.W. Samuel (idws@st-andrews.ac.uk) or Harald Haas (harald.haas@strath.ac.uk) ${ }^{1}$ LiFi Research and Development Centre, Department of Electronic \& Electrical Engineering, The University of Strathclyde, Technology \& Innovation Centre, 99 George Street, Glasgow G1 1RD, UK

${ }^{2}$ Organic Semiconductor Centre, SUPA, School of Physics and Astronomy, University of St Andrews, St Andrews KY16 9SS, UK

Full list of author information is available at the end of the article These authors contributed equally: Iman Tavakkolnia, Lethy K. Jagadamma, Rui Bian unprecedented data rates. It is reported in ref. ${ }^{2}$ that the IoT is expected to connect 500 billion smart devices by 2030. Currently, wireless access is essentially realized using the radio frequency (RF) spectrum. However, recent predictions made from available data and assumptions based on generally accepted forecasts show ${ }^{3}$ that there will not be a sufficient new RF spectrum for cellular communications by 2035 . Therefore, there is a need to explore other parts of the electromagnetic spectrum for wireless data access to combat the RF spectrum crunch. In this context, optical wireless communication (OWC) is of particular interest because it uses a vast and unregulated optical spectrum ${ }^{4}$. In particular, Light-Fidelity (LiFi), which is defined as a bidirectional, high-speed and fully networked OWC system, is a proven solution for mobile wireless networking ${ }^{5}$. Currently, there are global efforts to develop LiFi for future generations of wireless communication systems. To this end, LiFi is being standardized within the IEEE $802.11 \mathrm{bb}$ task group. The same standardization body, IEEE 802.11, has very successfully

\section{(c) The Author(s) 2021}

(c) (i) Open Access This article is licensed under a Creative Commons Attribution 4.0 International License, which permits use, sharing, adaptation, distribution and reproduction cc) in any medium or format, as long as you give appropriate credit to the original author(s) and the source, provide a link to the Creative Commons license, and indicate if changes were made. The images or other third party material in this article are included in the article's Creative Commons license, unless indicated otherwise in a credit line to the material. If material is not included in the article's Creative Commons license and your intended use is not permitted by statutory regulation or exceeds the permitted use, you will need to obtain permission directly from the copyright holder. To view a copy of this license, visit http://creativecommons.org/licenses/by/4.0/. 
standardized Wi-Fi. Digital data encoding in OWC systems is based on intensity modulation (IM) of light sources such as lasers or light-emitting diodes (LEDs). Consequently, data decoding is based on direct detection (DD) by one or more photodetectors (PDs). Different kinds of light sources and PDs have been used for OWC in a wide range of applications ${ }^{6-11}$. For instance, data rates as high as $15.7 \mathrm{~Gb} / \mathrm{s}$ have been demonstrated by the efficient utilization of inexpensive off-the-shelf LEDs and high-speed silicon PDs ${ }^{12}$.

Solar cells offer significant promise as high-speed data receivers, in addition to their main usage as energyharvesting devices, as previously demonstrated in ref. ${ }^{13,14}$, and more recently, data rates of up to $500 \mathrm{Mb} / \mathrm{s}$ from a single gallium arsenide (GaAs) solar cell have been reported by Fakidis et al. ${ }^{7}$. This dual function of photovoltaic (PV) systems is beneficially exploited for a wide variety of applications ranging from self-powered longrange free-space optical systems, where a large receiver exhibits significant advantages, to self-powered wearable devices as part of the future $\mathrm{IoT}^{15}$. Inorganic PVs, such as silicon ( $\mathrm{Si}$ ) and $\mathrm{GaAs}$, exhibit high power conversion efficiencies (PCEs) (>25\%) under solar spectrum illumination (air mass (AM) $1.5 \mathrm{G}$, corresponding to $100 \mathrm{~mW} /$ $\mathrm{cm}^{2}$ ). However, their use as optical receivers under indoor illumination is less efficient because the bandgaps of $\mathrm{Si}$ and GaAs (1.1 and $1.4 \mathrm{eV}$, respectively) are not well matched to the spectra of indoor lighting sources such as white LEDs and fluorescent lamps. This nonoptimal spectral overlap leads to a considerable drop in the PCE under indoor lighting conditions owing to carrier thermalization ${ }^{16,17}$. On the other hand, organic photovoltaics (OPVs) with conjugated organic semiconductors as the light-absorbing photoactive layer have an excellent spectral overlap with indoor artificial light. This leads to a 2-3 times enhanced PCE for OPVs compared with silicon solar cells, for which the efficiency drops (by two thirds) under indoor lighting ${ }^{17,18}$. Furthermore, OPVs are bandgap tuneable, printable, lightweight, flexible and amenable to roll-to-roll processing on customized and noncustomized substrates ${ }^{19,20}$. Therefore, powering a large portion of connected mobile IoT devices using flexible OPVs is an attractive solution ${ }^{21}$ to overcome the burden of having to charge devices regularly.

Apart from the recently reported $500 \mathrm{Mb} / \mathrm{s}$ data rate with GaAs inorganic $\mathrm{PVs}^{7}$, other works are mainly based on commercial silicon PVs with data rates below $20 \mathrm{Mb} / \mathrm{s}^{22-24}$. For instance, a solar panel model for simultaneous energy harvesting and data transmission was analyzed by Wang et al. ${ }^{14}$, and a data rate of $11.84 \mathrm{Mb} / \mathrm{s}$ was reported while harvesting $\sim 2 \mathrm{~mW}$ of electrical power. A self-reverse-biased solar panel optical receiver was presented by Shin et $\mathrm{al}^{25}$, showing a data rate of $17.05 \mathrm{Mb} /$ s. The promising potential of OPVs has also been previously demonstrated for two active layer blend structures of P3HT:PC ${ }_{61} \mathrm{BM}$ [poly(3-hexylthiophene) (P3HT) and phenyl C61-butyric acid methyl ester] and PTB7: $\mathrm{PC}_{71} \mathrm{BM}$ (poly[[4,8-bis[(2-ethylhexyl)oxy]benzo[1,2-b:4,5$\mathrm{b}^{\prime}$ ]dithiophene-2,6-diyl][3-fluoro-2-[(2-ethylhexyl)carbonyl]thieno[3,4-b]thiophenediyl]] (PTB7):[6,6]-phenylC ${ }_{71^{-}}$ butyric acid methyl ester $\left(\mathrm{PC}_{71} \mathrm{BM}\right)$ ) with data rates up to $50 \mathrm{Mb} / \mathrm{s}^{26,27}$. However, in each of these reports, the OPV device configuration was ITO/PEDOT:PSS/active layer/Ca or LiF/Al, where PEDOT:PSS (poly(3,4-ethylenedioxythiophene)-poly(styrenesulfonate)) is the hole extraction layer and calcium $(\mathrm{Ca})$ or lithium fluoride $(\mathrm{LiF})$ functions as the electron extraction layer. This standard structure suffers from severe performance instability and degradation issues accelerated by the inherent acidic and hygroscopic nature of PEDOT:PSS and the fast oxidation of low-workfunction electrodes such as calcium and aluminum ${ }^{28}$. However, performance stability is a crucial factor required for integrating OPVs into future applications. Previous studies have shown that an inverted device architecture, in which the polarity of charge collection is reversed, with metal oxide-based charge-selective transport layers and higher-work-function metal electrodes, such as gold/silver ( $\mathrm{Au} / \mathrm{Ag})$, has better performance stability than the standard structure $^{29}$. Hence, in this paper, OPVs with inverted device architectures are explored along with judiciously selected organic photoactive blend systems to combine a high data rate transmission capability with the known performance stability of the inverted structure. These types of OPVs have not been previously explored for OWC.

Multiple individual small OPVs can be incorporated into a single solar panel to harvest energy (a few milliwatts) for various applications. Furthermore, multiple light sources are usually installed in an indoor environment, and several LEDs are implemented in a single illumination device. This facilitates designing a multipleinput multiple-output (MIMO) structure based on OPVs. MIMO communication systems have been developed in theory and through experiments for several OWC applications $^{30}$. The merits and limitations of different techniques have been investigated ${ }^{31}$. An important benefit of an OPV-based MIMO system is that several independent data streams can be multiplexed to increase the overall data transmission rate while harvesting the aggregate energy of all individual solar cells. In addition, there is no need for electronics in the receiver to provide a negative bias voltage, and since the output is voltage, a transimpedance amplifier is not required. This may lead to simpler receiver circuitry. In fact, the analog electronics may only comprise passive electrical components ${ }^{14}$. The advantages especially unfold in an OPV-based MIMO system because it requires multiple receiver chains. Therefore, this novel system architecture can lead to an efficient solution for high data rate communication for 
future smart device applications. Note that the challenge in developing a MIMO-OWC system is interchannel cross-talk, which may limit the overall achievable data rate, and thus, cross-talk compensation methods may be necessary.

In this paper, we demonstrate a high bandwidth (i.e., $2.8 \mathrm{MHz}$ ) and record communication rates (i.e., $363 \mathrm{Mb} / \mathrm{s}$ ) from stable inverted OPVs. The organic photoactive layer is composed of a bulk heterojunction (BHJ) of the highly efficient polymer donor PTB7-Th (poly[4,8-bis(5-(2ethylhexyl)thiophen-2-yl)benzo[1,2-b4,5-b']dithiophene2,6-diyl-alt-(4-(2-ethylhexyl)-3-fluorothieno [3,4-b]thiophene-)-2-carboxylate-2-6-diyl]) with fullerene and nonfullerene acceptors ( $\mathrm{PC}_{71} \mathrm{BM}$ and $\left.\mathrm{EH}-\mathrm{IDTBR}\right)$, as shown in Fig. 1. The size of each OPV cell is $4 \mathrm{~mm}$ by $2.5 \mathrm{~mm}$. Under 1 Sun intensity $\left(100 \mathrm{~mW} / \mathrm{cm}^{2}\right)$, these solar cells demonstrate a PCE of $8.8 \%$, and under artificial indoor low-light-intensity illumination $\left(0.3-6 \mathrm{~mW} / \mathrm{cm}^{2}\right)$, the PCE is $14 \%$. To validate and assess the communication capabilities of these OPVs, a laser-based point-topoint OWC system is developed, and orthogonal frequency division multiplexing (OFDM) is incorporated to efficiently maximize the achievable data rate. The transmitted power of each laser is $56.2 \mathrm{~mW}$. A data rate of
$147.5 \mathrm{Mb} / \mathrm{s}$ is achieved with a single-input single-output (SISO) OPV-based system, which is the highest reported data rate with OPVs to the best of the authors' knowledge. In addition, $3.7 \mathrm{~mW}$ is simultaneously harvested by the same system. These values are further increased to $221 \mathrm{Mb} / \mathrm{s}$ or $363 \mathrm{Mb} / \mathrm{s}$ and $6.8 \mathrm{~mW}$ or $10.9 \mathrm{~mW}$ of harvested energy by implementing a 2-by-2 or 4-by-4 OPVbased MIMO system, respectively, for the first time. The presented MIMO structure can soon become a reality considering the rapid advancements in optical device technologies and MIMO communication techniques, which would enable reliable, bidirectional, energy-efficient, and cost-effective OWC.

\section{Results \\ OPVs}

OPVs were fabricated in the inverted configuration of ITO/ZnO/active layer $/ \mathrm{MoO}_{3} / \mathrm{Ag}$, as shown in Fig. 2a. The active layer was a $\mathrm{BHJ}$ of narrow bandgap donor polymers mixed with fullerene and nonfullerene acceptors. Although fullerenes are effective acceptors for charge separation in OPVs, their absorption in the visible light spectrum is poor. In the active layers of state-of-the-art highly efficient OPVs, the $\mathrm{PC}_{71} \mathrm{BM}$ fullerene acceptor is replaced by nonfullerene

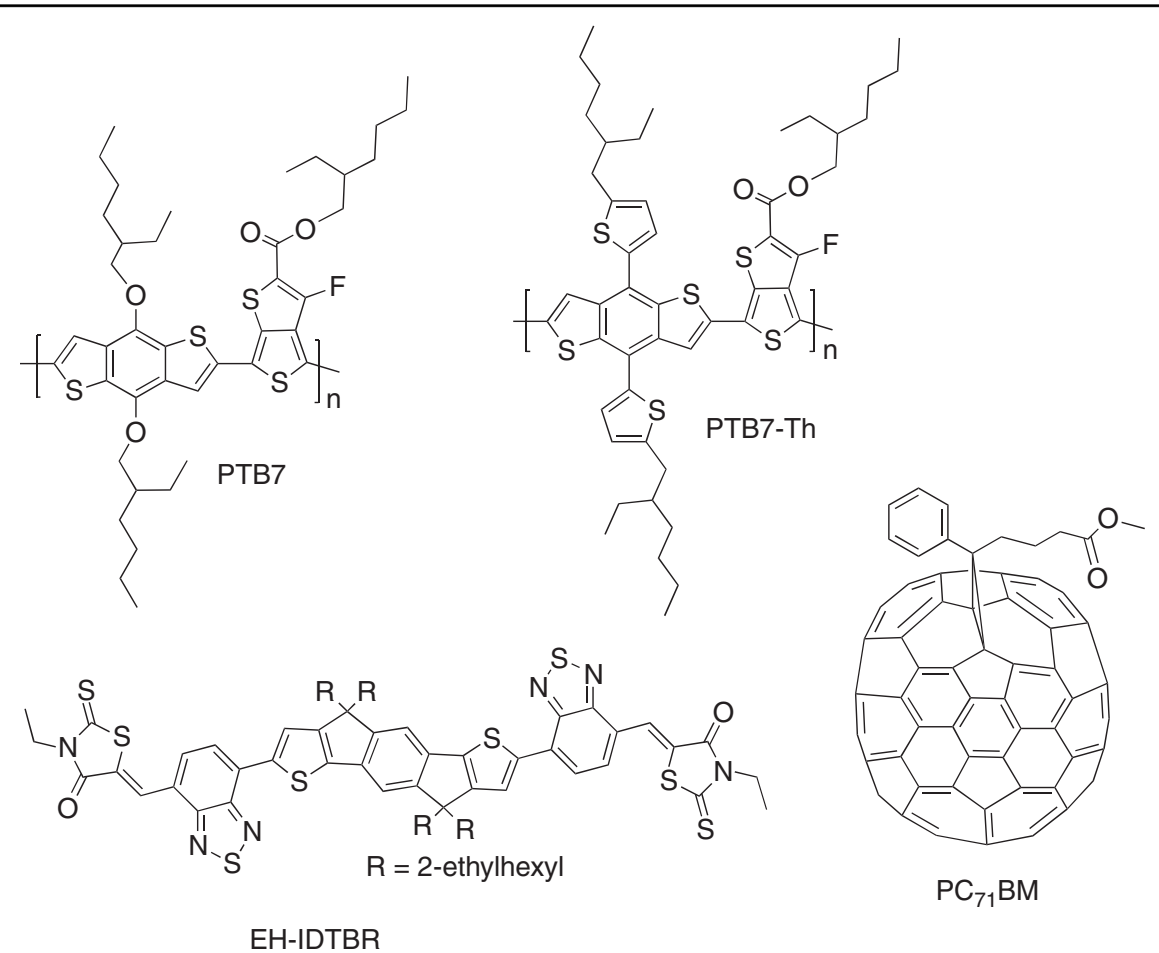

Fig. 1 Molecular structures of donor and acceptor molecules. PTB7 stands for the donor (poly[[4,8-bis[(2-ethylhexyl)oxy]benzo[1,2-b:4,5-b'] dithiophene-2,6-diyl][3-fluoro-2-[(2-ethylhexyl)carbonyl]thieno[3,4-b]thiophenediyl]]. PTB7-Th corresponds to the donor (poly[4,8-bis(5-(2-ethylhexyl) thiophen-2-yl)benzo[1,2-b4,5-b']dithiophene-2,6-diyl-alt-(4-(2-ethylhexyl)-3-fluorothieno [3,4-b]thiophene-)-2-carboxylate-2-6-diyl]). PC $C_{71} B M$ stands for the acceptor [6,6]-phenylC 71 -butyric acid methyl ester. EH-IDTBR corresponds to the acceptor 5,5'-[[4,4,9,9-tetrakis(2-ethylhexyl)-4,9-dihydro-sindaceno[1,2-b:5,6-b']dithiophene-2,7-diyl]bis(2,1,3-benzothiadiazole-7,4-diylmethylidyne)]bis[3-ethyl-2-thioxo-4-thiazolidinone]. 

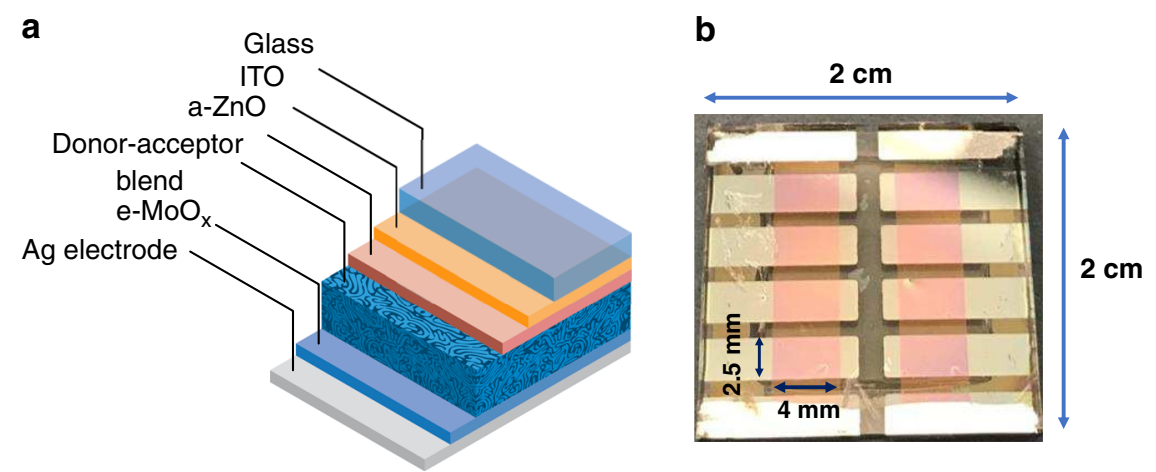

Fig. 2 OPV design. a Schematic of the inverted OPV device architecture; ITO: indium tin oxide, a-ZnO: amorphous zinc oxide, e-MoOx: evaporated molybdenum oxide, Ag: element silver. b Photograph of the fabricated OPV sample. It shows eight individual cells (red squares) and four common ground pads at the corners.

acceptors such as EH-IDTBR, with higher absorption in the visible range and thus higher efficiency ${ }^{32}$. The donor polymer in the present investigation was selected based on its high PCE and the high open-circuit voltage, $V_{\mathrm{oc}}=1 \mathrm{~V}$, under 1 Sun $\left(100 \mathrm{~mW} / \mathrm{cm}^{2}\right)$ when blended with the nonfullerene acceptor used here. The narrow absorption band of OPVs with a high open-circuit voltage, $V_{\mathrm{oc}}>1 \mathrm{~V}$, is beneficial for indoor low-intensity lighting conditions, as the drop in $V_{\mathrm{oc}}$ with light intensity will be smaller than that for silicon solar cells ${ }^{33-35}$. In the present work, the simultaneous energy harvesting and data detection properties of traditional and state-of-the-art inverted OPVs constituted by both fullerene- and nonfullerene-based $\mathrm{BHJ}$, such as PTB7:PC 71 BM, PTB7-Th:PC 71 BM, and PTB7-Th:EHIDTBR, are investigated. The architecture of the OPV device structure is shown in Fig. 2a, and the actual fabricated solar cells are shown in Fig. $2 \mathrm{~b}$. There are eight individual solar cells (the red areas) on a $2 \mathrm{~cm}$ by $2 \mathrm{~cm}$ substrate and four common ground pads at the corners. The eight solar cells have separate contacts so that the output current from each of them is individually accessible. Therefore, the individual cells can be used as separate receivers, as in the current work, or their signals can be combined so that the whole panel acts as one receiver.

The characteristics of these solar cells were tested under both solar spectrum and indoor lighting illumination. The results are presented in the following subsections. The emission spectra of different illumination sources are demonstrated in Supplementary Information, Fig. S1.

\section{1) OPV performance-solar spectrum illumination}

The absorption spectra of the three different OPV blend systems were measured and are presented in the Supplementary Information, Fig. S2. The absorption spectra of the PTB7:PC ${ }_{71} \mathrm{BM}$ and PTB7-Th:PC $\mathrm{P}_{71} \mathrm{BM}$ blends span $350 \mathrm{~nm}-750 \mathrm{~nm}$, whereas for the PTB7-Th: EH-IDTBR blend, a narrower absorption spectrum of
$550 \mathrm{~nm}-750 \mathrm{~nm}$ in the visible light spectrum is observed. The PCEs of the OPV cells were determined from their current density-voltage $(J-V)$ characteristics under $100 \mathrm{~mW} / \mathrm{cm}^{2}$ light intensity from a solar simulator at a distance of $40 \mathrm{~cm}$. The $J-V$ characteristics and the external quantum efficiency (EQE) of each blend system are shown in Fig. 3, and the PV performance parameters are presented in the Supplementary Information, Table S1. PTB7:PC ${ }_{71}$ BM demonstrates a PCE of $\sim 7.6 \%$, and the PTB7-Th-based BHJs exhibit a higher PCE of $\sim 8.7 \%$, which can be attributed to the narrower bandgap of PTB7-Th compared with PTB7, which is reflected in its larger short-circuit current density $J_{\mathrm{sc}}$. The PCE of the nonfullerene acceptor BHJ (PTB7-Th: EH-IDTBR) is $\sim 8.8 \%$, and it has a higher $V_{\text {oc }} \sim 1 \mathrm{~V}$ than the fullerene-based $\mathrm{BHJ}$ solar cells. The high $V_{\mathrm{oc}}$ of the OPVs with BHJs based on a nonfullerene acceptor can be attributed to the reduced energy loss via nonradiative recombination during exciton dissociation and generation of charge carriers ${ }^{36-38}$.

\section{2) OPV performance-indoor lighting conditions}

In addition to the performance evaluation of the PTB7-Th-based solar cells under 1 Sun intensity, their performance under indoor lighting conditions was also measured. Indoor lighting in residential buildings and offices is dominated by fluorescent lights and white LEDs, which are significantly different from the solar spectrum in intensity and spectral content. Under white LED lighting with an illumination intensity of $5.9 \mathrm{~mW} /$ $\mathrm{cm}^{2}$, the PCE is $11 \%$ for PTB7-Th:PC ${ }_{71} \mathrm{BM}$ with an output power of $0.64 \mathrm{~mW} / \mathrm{cm}^{2}$ and $14 \%$ for PTB7-Th: EH-IDTBR with an output power of $0.83 \mathrm{~mW} / \mathrm{cm}^{2}$ (Table S2). The corresponding $J-V$ characteristics are shown in Fig. 4a. The higher PCE of the PTB7-Th:EHIDTBR blend compared with PTB7-Th:PC ${ }_{71} \mathrm{BM}$ mainly originates from the higher $V_{\mathrm{oc}}$ and $J_{\mathrm{sc}}$ under indoor 

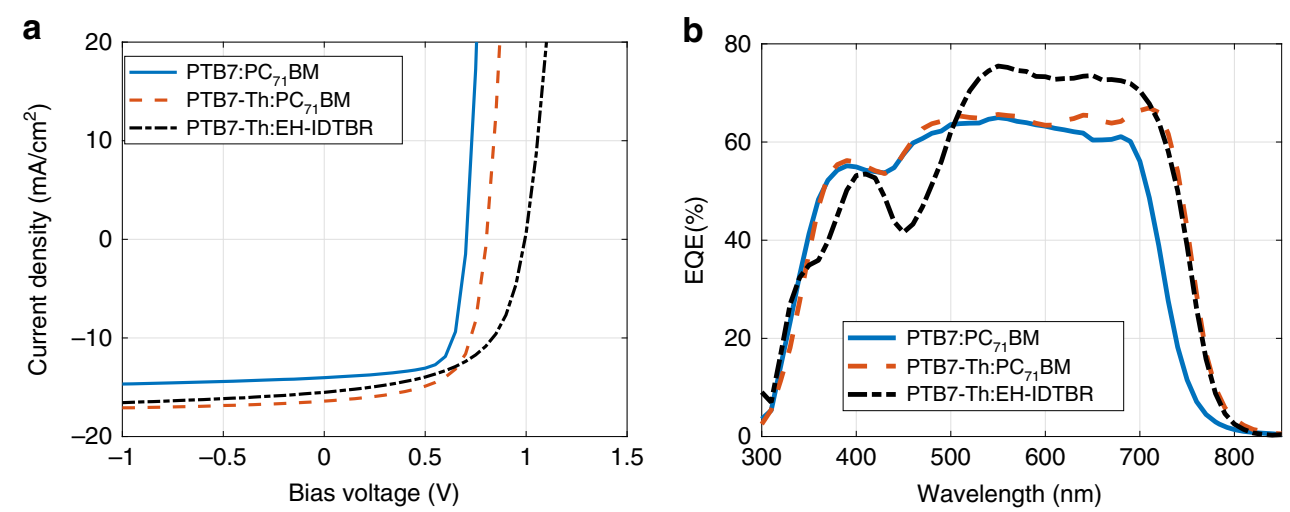

Fig. 3 Performance of OPV cells under solar spectrum illumination. a $J-V$ characteristics of OPV cells are shown corresponding to three different BHJ blends of PTB7:PC 71 BM, PTB7-Th:PC 71 BM and PTB7-Th:EH-IDTBR, measured under $100 \mathrm{~mW} / \mathrm{cm}^{2}$ light intensity from a solar simulator. $\mathbf{b}$ EQE spectra of the corresponding OPV cells.
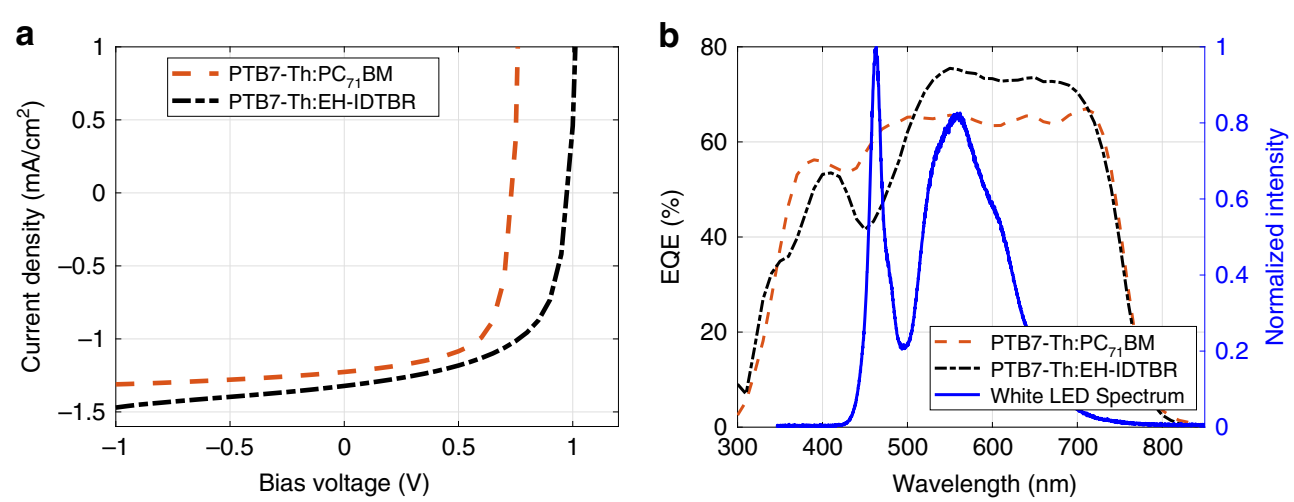

Fig. 4 Performance of OPV cells under indoor lighting. a $J-V$ characteristics of OPV cells are shown corresponding to BHJ blends of PTB7-Th: PC $C_{71} B M$ and PTB7-Th:EH-IDTBR under white LED illumination. $\mathbf{b}$ Graph showing the spectral overlap of the EQE of the PTB7-Th:PC 71 BM and PTB7-Th: EH-IDTBR blends (left axis) with the spectrum of the white LED (right axis).

lighting conditions. This can be attributed to the higher EQE of the PTB7-Th:EH-IDTBR blend for the emission spectra of white LEDs, as shown in Fig. 4b. This reduces the carrier loss from thermalization and non-absorption. The PV performance of the PTB7-Th:EH-IDTBR blend under fluorescent lighting, with an illumination intensity of $0.7 \mathrm{~mW} / \mathrm{cm}^{2}$, was also tested, and a PCE of $13.6 \%$ was obtained with an output power of $95 \mu \mathrm{W} / \mathrm{cm}^{2}$. A comparison of the PV properties of our best-performing OPV blend, PTB7-Th:EH-IDTBR, under 1 Sun and indoor illumination is shown in Fig. S4. Compared with 1 Sun illumination, the PCE is considerably improved under indoor illumination, and $80 \%$ of $V_{\mathrm{oc}}$ is retained along with a slightly improved fill factor. The demonstrated high PCE of the OPVs affirms their promising capability for indoor light energy-harvesting applications. In addition, the EQE spectra of commercial solar cells, such as silicon and GaAs, are compared with those of our OPVs in Supplementary Note 1.

\section{Communication protocols}

The availability of a safe and affordable spectrum has become a crucial factor of wireless systems. Although there is a vast amount of free and safe spectrum in the visible light domain, the performance of visible light communication (VLC) is generally limited by the bandwidth of optical components, such as LEDs and PDs. The slow response of these optical components in electrical-optical signal conversion causes this bandwidth constraint. In an OPV-based system, the system bandwidth is limited by OPV cells to a few MHz. Therefore, it is important to optimize the system to obtain the highest possible data rate with this bandwidth constraint. As the system is designed based on single-sided baseband modulation, the maximum frequency used for signal modulation directly determines the achievable data rate of the system.

OFDM is an efficient signaling scheme by means of which a data rate close to the theoretical capacity limit of the communication channel can be achieved. It has been 


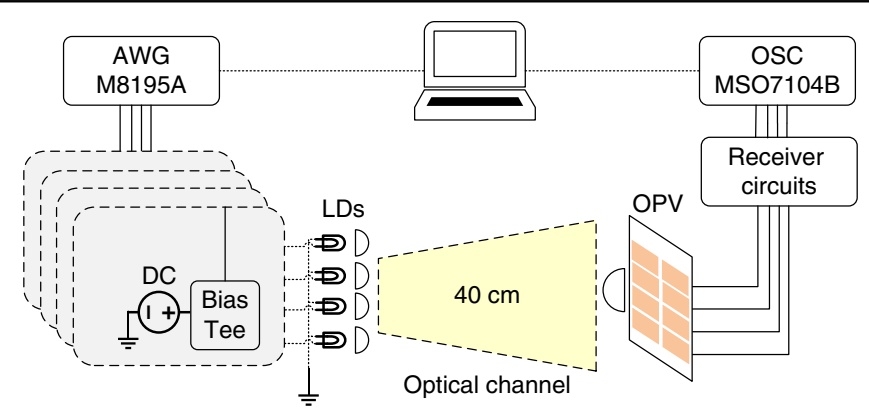

Fig. 5 Block diagram of the system. A digital signal is generated by a computer and sent to an arbitrary waveform generator (AWG) for digital-toanalogue conversion. The analogue signal is combined with a direct current (DC) bias using a bias tee. Depending on the experiment format, one, two, or four laser diodes (LDs), mounted in lens tubes with an adjustable focal length, are used to transmit the optical signals. One large lens is used at the receiver to focus the light beams on the required number of OPV cells. The receiver circuit is used to extract the signal and harvest the DC power. Details of the receiver circuit are provided in Supplementary Information. An oscilloscope (OSC) is used to convert the analogue signal to a digital signal and send it to a computer for processing. More details of the setup are provided in the Materials and methods section.

proven in practice that OFDM can deliver high data rate communication and, at the same time, provide relatively simple solutions for some of the most challenging problems in wireless communication, such as the frequency selectivity of the wireless channel that could cause severe intersymbol interference ${ }^{39}$. OFDM was first developed for RF communication, where signals are bipolar and complex. However, some modifications are necessary to make an OFDM signal real and positive, which is suitable for IM/DD systems in $\mathrm{VLC}^{5,40}$. In addition, by using adaptive bit and power loading, it is straightforward to maximize the data rate in a VLC system that usually exhibits a lowpass frequency-selective response ${ }^{41}$. The available signalto-noise ratio (SNR) determines the modulation depth of each subcarrier at a specific target bit error ratio (BER), and as a result, the total achievable data rate can be estimated $^{42}$.

Direct current biased optical OFDM (DCO-OFDM) is an efficient and low-complexity method used in VLC to achieve high data rates ${ }^{6,12,40}$. By imposing Hermitian symmetry on the signal vector in the frequency domain, a real-time domain signal is obtained after the Fourier transformation. Then, a constant direct current (DC) bias is added to the signal so that it becomes positive. However, depending on the value of the DC bias, a few samples may still be negative, which are clipped to zero before transmission. Taking into account the Hermitian symmetry and the DC bias (i.e., no data can be modulated in subcarriers $\mathrm{X}_{0}$ and $X_{N_{\mathrm{s}} / 2}$ ), the frequency-domain subcarrier vector, $\mathbf{X}$, is described as follows:

$$
\mathbf{X}=\left[0, X_{1}, \ldots, X_{\frac{N_{\mathrm{s}}}{2}-1}, 0, X_{\frac{N_{\mathrm{s}}}{2}-1}^{*}, \ldots, X_{1}^{*}\right]
$$

where $N_{\mathrm{s}}$ is the number of available subcarriers. In (1), $X_{k}, 1 \leq k \leq \frac{N_{s}}{2}-1$, are the modulated data symbols at each subcarrier, $k$. Each subcarrier is modulated by an $M_{k^{-}}$ ary quadrature amplitude modulation signal. The signal constellation for each subcarrier may be different, hence the use of the subscript $k$. The value of $M_{k}$ depends on the available SNR at the respective subcarrier. In this paper, based on the adaptive bit loading algorithm ${ }^{41}$, the maximum $M_{k}$ is found iteratively for the available SNR at each subcarrier so that the BER is below the target BER of $4.7 \times 10^{-3}$. Error-free data transmission is then feasible using a forward error correction algorithm with $6.25 \%$ coding overhead ${ }^{43}$. More details are presented in Supplementary Note 2.

Signal multiplexing in the spatial domain is an effective solution to increase the achievable data rate in VLC systems with multiple inputs and multiple outputs. Space constitutes an extra degree of freedom in addition to the time and frequency dimensions. Spatial multiplexing is a MIMO technique and is particularly suitable for an OPV-based system since multiple cells are available at the receiver side to form a panel and the additional hardware complexity for MIMO implementation is minimal. The challenge is to manage and reduce the cross-talk between different links. The cross-talk results in an interference signal, which, similar to thermal noise, reduces the total system capacity. In an OPV-based system, interference in the optical domain can be eliminated almost completely by using appropriate lenses that allow light beam alignment to specific individual receiver cells. This is also referred to as imaging MIMO. However, interference in the electrical domain is unavoidable in practical scenarios because of the dual use of the OPV panel as an energy-harvesting device and a communication device. To be able to aggregate the energy of multiple OPV cells, they are usually connected either in series or in parallel. In other words, this is a one-port system. In contrast, it is necessary to extract signals from each OPV cell individually for MIMO operation. This requires a multi-port system. As a consequence, a circuit design is required that aggregates $\mathrm{DC}$ power while at the same time 
enabling extraction of alternating current (AC) signals from each OPV cell. This issue inevitably causes interference in the electrical domain. We note that while it is straightforward to improve the harvested energy by increasing the number of OPV cells, this poses an increasing challenge for interference mitigation for efficient MIMO communication.

\section{Proof-of-principle experiments for OPV-based communication}

In this section, experimental results are presented that demonstrate the feasibility of simultaneous high-speed data transmission and efficient energy harvesting by multiple OPVs. The communication performance of specially fabricated OPVs is investigated by the experimental setup shown in Fig. 5. A single light source and a single OPV cell are used for the SISO setup, whereas two and four of each are used for the 2-by-2 and 4-by-4 MIMO experiments, respectively. Random data generation and data detection from the received signal are processed offline by means of a computer using MATLAB software. The details of the setup shown in Fig. 5 are given in the Materials and methods section.

A summary of the results for the different types of OPVs is presented in Table 1 . A data rate of $\rho=147.5 \mathrm{Mb} / \mathrm{s}$ and a harvested power of $E_{\mathrm{H}}=3.7 \mathrm{~mW}$ are obtained using

Table 1 Results for energy harvesting and data transmission for SISO experiments.

\begin{tabular}{llll}
\hline OPV blend & $\boldsymbol{E}_{\mathbf{H}}[\mathrm{mW}]$ & $\boldsymbol{\rho}[\mathrm{Mb} / \mathbf{s}]$ & BER \\
\hline PTB7:PC 71 BM & 3.3 & 90.3 & $3.6 \times 10^{-3}$ \\
PTB7-Th:PC 71 BM & 3.5 & 78.4 & $1.6 \times 10^{-3}$ \\
PTB7-Th:EH-IDTBR & 3.7 & 147.5 & $2.8 \times 10^{-3}$ \\
\hline
\end{tabular}

$E_{H}$ Harvested power, $\rho$ data rate.
PTB7-Th:EH-IDTBR. The achieved data rate is the highest reported for a SISO setup when OPVs are used as receivers compared with previously published data rates using OPVs ${ }^{26,27}$. Estimated and measured SNR values are presented in Fig. 6a. Moreover, the bit loading results are shown in Fig. 6b. Note that these results correspond to the best single cell among the eight available cells of the OPV panel. The recorded BERs are below the harddecision forward error correction coding threshold of $4.7 \times 10^{-3}$, which means that with a $6.25 \%$ coding overhead, essentially error-free communication is possible ${ }^{43}$. The measured $-3 \mathrm{~dB}$ bandwidths of the OPVs are 1.32 MHz, $1.26 \mathrm{MHz}$, and $2.77 \mathrm{MHz}$ for PTB7:PC ${ }_{71} \mathrm{BM}$, PTB7-Th:PC ${ }_{71}$ BM, and PTB7-Th:EH-IDTBR, respectively. The channel responses of the OPVs are presented in the Supplementary Information, Fig. S6. A total of 512 OFDM subcarriers were used. Owing to the adaptive bit and power loading used, the modulation bandwidths for PTB7:PC ${ }_{71}$ BM, PTB7-Th:PC ${ }_{71}$ BM, and PTB7-Th:EHIDTBR were $19.48 \mathrm{MHz}, 17.52 \mathrm{MHz}$, and $30.1 \mathrm{MHz}$, respectively, which are considerably higher than the $-3 \mathrm{~dB}$ bandwidths.

Note that the light spectrum of the laser diode (LD) used in this experiment is part of the indoor white light emission spectrum. White light with the desired temperature can be produced by adding blue and green light sources. As a consequence, wavelength division multiplexing (WDM) can be implemented to enhance the data rates ${ }^{12}$. We focus on the single-color transmission here and leave the inclusion of WDM for future work. In the following, the MIMO system is presented to signify the potential of using OPVs as optical receivers for future VLC applications.

For the 2-by-2 MIMO setup, two equivalent LDs and two OPV cells on the same panel were used. The transmitters, LDs (Thorlabs, 6544FM) mounted in lens tubes with an adjustable focal length (Thorlabs, LT230220P-B, $5.6 \mathrm{~mm}$ diameter), were oriented towards the receiver
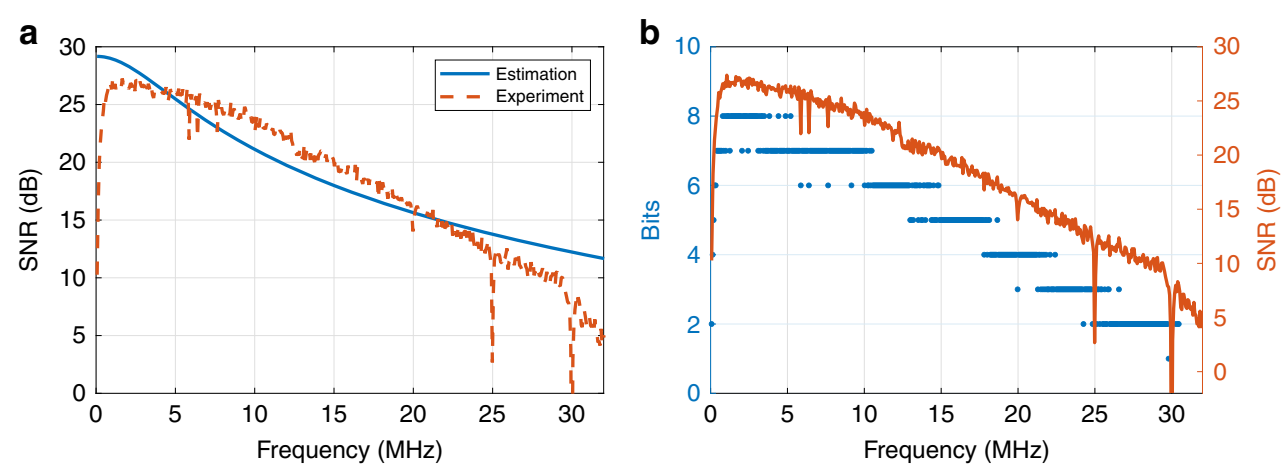

Fig. 6 Communication performance of the PTB7-Th:EH-IDTBR blend OPV cell. a The estimated and measured SNR values are shown for the best single cell among the eight available cells of the OPV panel. $\mathbf{b}$ The number of bits loaded onto each subcarrier channel (left axis) and the measured SNR values (right axis). A greater number of bits is allocated to subcarriers that experience a higher available SNR. This is referred to as adaptive bit loading. 

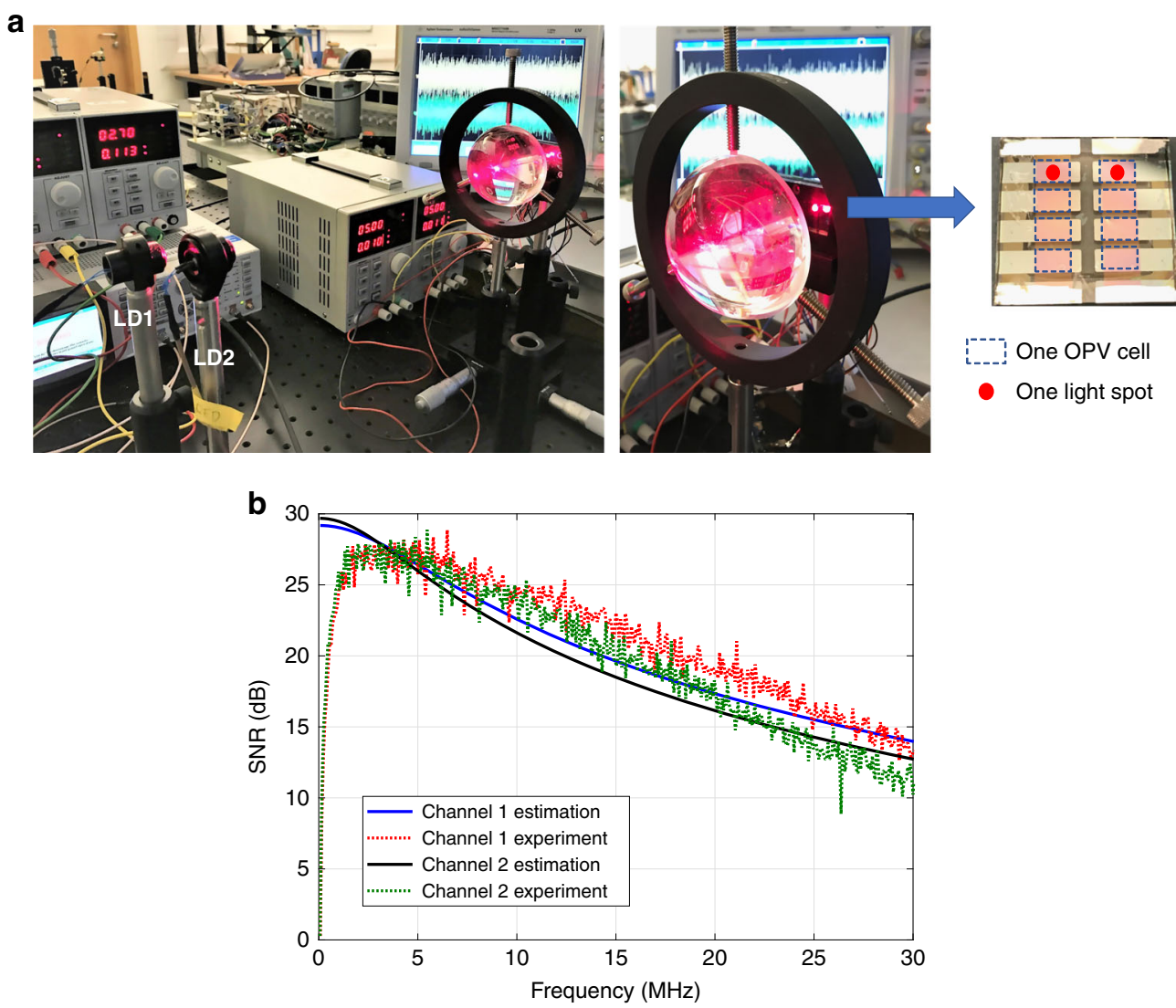

Fig. 7 Proof-of-concept of MIMO experiment. a Photograph of the 2-by-2 MIMO setup in the lab (left). Two laser diodes (LDs) are used to transmit the signals. One large aspheric lens is used at the receiver to focus the light beams on two separate OPV cells (right). $\mathbf{b}$ Estimated and measured SNR values for two channels of the 2-by-2 MIMO experiment.

Table 2 Results for energy harvesting and data transmission for MIMO.

\begin{tabular}{lllll}
\hline OPV blend & MIMO & $E_{\mathrm{H}}[\mathrm{mW}]$ & $\boldsymbol{\rho}[\mathrm{Mb} / \mathbf{s}]$ & BER \\
\hline PTB7:PC 71 BM & 2 -by-2 & 5.8 & 122 & $3.5 \times 10^{-3}$ \\
PTB7-Th:EH-IDTBR & $2-$-by-2 & 6.8 & 221 & $3.4 \times 10^{-3}$ \\
PTB7-Th:EH-IDTBR & $4-$-by-4 & 10.9 & 363 & $1.1 \times 10^{-3}$ \\
\hline
\end{tabular}

$E_{\mathrm{H}}$ Harvested power, $\rho$ data rate.

lens. Light beams from the two LDs were each focused on one of the cells by a single lens (Thorlabs, ACL7560, $75 \mathrm{~mm}$ diameter). The distance between the receiver lens and the OPV panel was manually adjusted until two clear spots were observed on two separate OPV cells, and consequently, two parallel optical channels were generated in the spatial domain. This allowed us to have links with negligible optical cross-talk. A picture of the setup is shown in Fig. 7a, where the two light spots originating from two laser sources can be seen. The spot sizes were adjusted to fit within each OPV cell. The size of the OPV panel, which consists of eight cells, is $2 \mathrm{~cm}$ by $2 \mathrm{~cm}$, and each cell is $4 \mathrm{~mm}$ by $2.5 \mathrm{~mm}$ in size; thus, the spot size is roughly a circle with a diameter of $2.5 \mathrm{~mm}$. For the MIMO experiments, subsets of the eight OPV cells were chosen. Although the optical channel matrix was almost diagonal, the electrical channel and consequently the overall channel matrices were nondiagonal due to the structure of the receiver circuit. Details are presented in Supplementary Notes 3 and 4. Therefore, there is some crosstalk in the electrical domain among the received signals from each OPV cell. To mitigate this cross-talk effect, zero forcing (ZF) was used to demultiplex the desired signal of each channel. ZF was performed by multiplying the received signal in vector format by the inverse of the channel matrix. Details are presented in the Methods section. The estimated and measured SNR values are demonstrated in Fig. 7b. Measurements were taken using PTB7-Th:EH-IDTBR OPVs. The results are shown in Table 2. The achieved data rates for the PTB7:PC ${ }_{71} B M$ and PTB7-Th:EH-IDTBR OPVs are $122 \mathrm{Mb} / \mathrm{s}$ and $221 \mathrm{Mb} / \mathrm{s}$, respectively, whereas the measured harvested powers are $5.8 \mathrm{~mW}$ and $6.8 \mathrm{~mW}$, respectively. Note that the minimum mean square error algorithm was also used 
as an alternative demultiplexing technique. However, the results were almost the same due to the specific channel matrix values and relatively high SNRs at the utilized subcarriers. Therefore, only results using ZF are reported. A 4-by-4 MIMO experiment was also set up and tested using four red LDs and four OPV cells of type PTB7-Th: EH-IDTBR. The results are shown in Table 2. A record data rate of $363 \mathrm{Mb} / \mathrm{s}$ is achieved using four OPVs. We note that the capacity per channel decreases for a larger number of cells due to the increased interference.

\section{Discussion}

This paper reports a fundamental breakthrough in the capability of OPVs to function as both energy-harvesting devices and high-speed data detectors. We achieved this by jointly advancing PV materials, device structures, and communication techniques. The OPVs used in this work exhibit a $-3 \mathrm{~dB}$ bandwidth as high as $2.77 \mathrm{MHz}$, which is the highest ever reported. Furthermore, the OPVs demonstrate a high power conversion efficiency of 13-14\% under indoor lighting conditions. For PTB7-Th:EHIDTBR-type OPVs, a data rate of $147.5 \mathrm{Mb} / \mathrm{s}$ (i.e., three times faster than the previous single OPV device) was achieved for a SISO system. We used a MIMO technique consisting of a maximum of four LDs and four OPVs to increase the data rate to a remarkable value of $363 \mathrm{Mb} / \mathrm{s}$. With the four OPV cells, we simultaneously extracted a power of $10.9 \mathrm{~mW}$.

Although the reported results are for a specific system configuration of four light transmitters and four OPV cells integrated into a single panel, the reported methodology is generally applicable. As a result, by using the proposed method, it is possible to trade the physical size of the system for both harvested energy and data rate.

OPVs are very attractive because they are easily made and can be flexible, thereby allowing their integration into internet-connected devices. In addition, compared with inorganic detectors, OPVs have the potential to be significantly cheaper, which is a key driver of their large-scale commercial adoption. Although the demand for wireless data transmission capacity is ever increasing, VLC provides unregulated, safe, and vast resources to alleviate emerging wireless capacity bottlenecks. The additional feature of being able to directly harvest the energy from the data-carrying signal could be used to enhance the battery life of mobile devices, which is one of the key issues of modern wearables.

\section{Materials and methods}

\section{Fabrication and testing of organic solar cells}

Inverted organic solar cells were fabricated on prepatterned ITO-coated glass. The ITO-coated glass substrates were cleaned in detergent (sodium dodecyl sulfate), successively ultrasonicated in deionized water, acetone, and isopropyl alcohol, and exposed to oxygen plasma for $3 \mathrm{~min}$. PTB7: $\mathrm{PC}_{71} \mathrm{BM}$ blend solutions were prepared by dissolving the components in a ratio of 1:1.5 (by weight), with a total concentration of $25 \mathrm{mg} / \mathrm{mL}$ in chlorobenzene, with 3 vol\% DIO. In the case of the PTB7$\mathrm{Th}: \mathrm{PC}_{71} \mathrm{BM}$ blend, the donor:acceptor components were weighed in a ratio of $1: 1.5$, with a total concentration of $25 \mathrm{mg} / \mathrm{mL}$ in ortho-dichlorobenzene solvent. For the PTB7-Th:EH-IDTBR blend system, the active layer solution was prepared by blending the donor-acceptor components in a 1:1 weight ratio, with a total concentration of $20 \mathrm{mg} / \mathrm{mL}$ in ortho-dichlorobenzene solvent. For all the inverted organic solar cells fabricated, the electron transporting layer was a thin film of amorphous $\mathrm{ZnO}(\mathrm{a}-\mathrm{ZnO})$ with a thickness of $\sim 25 \mathrm{~nm}$ and was prepared following the method used by Jagadamma et al. ${ }^{44}$. The active layer was prepared by spin coating on glass/ ITO/a- $\mathrm{ZnO}$ substrates inside a nitrogen-filled glove box as follows: for the PTB7: $\mathrm{PC}_{71} \mathrm{BM}$ blend, the spin-coating condition was $1500 \mathrm{rpm}$ for $60 \mathrm{~s}$; for the PTB7-Th: $\mathrm{PC}_{71} \mathrm{BM}$ layer, spin coating was performed at $1200 \mathrm{rpm}$ for $60 \mathrm{~s}$; and for PTB7-Th:EH-IDTBR, the spin-coating process was carried out at $900 \mathrm{rpm}$ for $60 \mathrm{~s}$. The samples were then transferred to a vacuum thermal evaporator $\left(1 \times 10^{-6}\right.$ mbar base pressure $)$ and kept under vacuum overnight before thermally evaporating the hole transporting layer of $\mathrm{MoOx}(4 \mathrm{~nm})$ and anode of $\mathrm{Ag}(100 \mathrm{~nm})$ using a shadow mask. The aperture area of the shadow mask used for the measurement of the OPV devices was $0.065 \mathrm{~cm}^{2}$. After electrode deposition, the devices were encapsulated with a UV optical adhesive and a glass coverslip. The current-voltage characteristics were determined under an illumination intensity of $100 \mathrm{~mW} /$ $\mathrm{cm}^{2}$ in the air using an AM 1.5 global Sciencetech (SS150 -AAA) solar simulator at a distance of $40 \mathrm{~cm}$ and a Keithley 2400 source-measure unit. The illumination intensity was verified with a calibrated monosilicon detector and a KG-5 filter. The EQE measurements were performed at zero bias by illuminating the device with monochromatic light supplied from a xenon arc lamp in combination with a dual-grating monochromator. The number of photons incident on the sample was calculated for each wavelength by using a silicon photodiode calibrated by the National Physical Laboratory. For the indoor measurements, the LED light source used was a Cree XML T6, and the illumination intensity $(5.9 \mathrm{~mW} /$ $\mathrm{cm}^{2}$ ) was adjusted by changing the input voltage at a distance of $4 \mathrm{~cm}$. A fluorescent lamp (RS components, PL 11 lamp, $11 \mathrm{~W}$ ) was also used as an illumination source $\left(0.7 \mathrm{~mW} / \mathrm{cm}^{2}\right)$ at a distance of $30 \mathrm{~cm}$. The irradiance level was measured using an RK 5710 power radiometer and an Optometer P9710. The absorption spectra of the active layer blends were recorded using a CARY $300 \mathrm{UV}$ Visible Spectrophotometer. 


\section{SNR measurement and estimation}

Prior to the data transmission, several training sequences were transmitted by each light source, one at a time for the MIMO case. A sequence of known quadrature phase-shift keying-modulated symbols was used in a DCO-OFDM transmission scheme. This allowed for accurate estimation of the frequency response as well as the channel gain at each subcarrier. First, channel gains at different frequencies were measured and averaged over 80 OFDM frames. Then, the noise power was estimated as the difference between the total received power and the noise-free received signal power, which was determined based on the measured channel gain and the known training sequence. For the SISO case, the SNR was estimated as the ratio of the noise-free received signal power and noise power. Note that the sample sequence generated in MATLAB software was assumed to be the input signal, and the sample sequence captured by the oscilloscope after the digital-to-analog conversion was assumed to be the output signal. Therefore, the total channel response, including the effects of all components, was determined.

For the MIMO scenario, the communication channel at the $k^{\text {th }}$ subcarrier is an additive white Gaussian noise channel. The system model for $N_{\mathrm{t}}$ transmitters and $N_{\mathrm{r}}$ receivers can therefore be written as follows:

$$
\mathbf{y}_{k}^{N_{\mathrm{r}} \times 1}=\mathbf{H}_{k}^{N_{\mathrm{r}} \times N_{\mathrm{t}}} \mathbf{x}_{k}^{N_{\mathrm{t}} \times 1}+\mathbf{n}_{k}^{N_{\mathrm{r}} \times 1}
$$

where vectors $\mathbf{y}_{k}^{N_{\mathrm{r}} \times 1}$ and $\mathbf{n}_{k}^{N_{\mathrm{r}} \times 1}$ are the received signal and noise, respectively. The channel matrix $\mathbf{H}_{k}^{N_{\mathrm{r}} \times N_{\mathrm{t}}}=$ $\mathbf{G}_{k}^{N_{\mathrm{r}} \times N_{\mathrm{r}}} \mathbf{H}_{0}^{N_{\mathrm{r}} \times N_{\mathrm{t}}}$ is the total channel matrix at the corresponding subcarrier, where the matrices $\mathbf{G}_{k}^{N_{\mathrm{r}} \times N_{\mathrm{r}}}$ and $\mathbf{H}_{0}^{N_{\mathrm{r}} \times N_{\mathrm{t}}}$ represent the electrical and optical channel gains, respectively. The vector $\mathbf{x}_{k}^{N_{\mathrm{t}} \times 1}$ refers to the transmitted signal at the $k^{\text {th }}$ subcarrier. Elements of the channel matrix are estimated as previously described and can be calculated using existing models ${ }^{28}$. The effective noise consists of thermal noise and shot noise and is assumed to be white Gaussian $\mathcal{N}\left(0, \sigma^{2} \mathbf{I}_{N_{\mathrm{r}}}\right)$, where $\mathbf{I}_{N_{\mathrm{r}}}$ is an ideal matrix of size $N_{\mathrm{r}}$. To remove the cross-talk between channels, ZF was applied by multiplying the received vector by the inverse of the channel matrix $\mathbf{y}_{k} \mathbf{H}_{k}^{-1}$. Then, the SNR was estimated in the same way as described for SISO. The SNR at the $k^{\text {th }}$ subcarrier can also be calculated as ${ }^{43}$

$$
\gamma_{k, n}^{\mathrm{ZF}}=\frac{P_{\text {elec }}^{k}}{\sigma^{2}\left[\left\{\mathbf{H}_{k}^{\dagger} \mathbf{H}_{k}\right\}^{-1}\right]_{n n}}
$$

where $P_{\text {elec }}^{k}$ is the total electrical input signal power at the $k^{\text {th }}$ subcarrier, + denotes the matrix conjugate transpose, and []$_{n n}$ denotes the $n^{\text {th }}$ diagonal element.

\section{Data transmission measurement}

A red LD (Thorlabs, HL6544FM) with a dominant wavelength of $660 \mathrm{~nm}$ was used as the light source. The DC bias was provided by the bench power supply (TENMA 7210505). The OFDM signal was generated by a laptop using MATLAB and fed to the LD by an arbitrary waveform generator (AWG, Keysight M8195A). The OFDM signal and DC bias were combined with a bias tee (Mini-Circuit, ZFBT-4R2GW+). Each transmitter (Thorlabs, 6544FM) was mounted in a lens tube with an adjustable focal length (Thorlabs, LT230220P-B, $5.6 \mathrm{~mm}$ diameter). For the 2-by-2 MIMO setup, the two LD modules were arranged side by side with a distance of $2.5 \mathrm{~cm}$ between them. At the receiver, a single aspheric condenser lens (Thorlabs, ACL7560, $75 \mathrm{~mm}$ diameter) was used. The link distance was set to $40 \mathrm{~cm}$. On the receiver end, the fabricated OPV panels shown in Fig. 2 were used, and the output from each OPV cell was connected to a custom-designed receiver circuit that contained two branches for signal detection and energy harvesting. A capacitor and a load resistor $(50 \Omega)$ were incorporated into the signal detection branch of the circuit. The energy-harvesting branch contained an inductor and another load resistor $(50 \Omega)$. The LD was driven at $100 \mathrm{~mA}$, and the measured output optical power was $56.2 \mathrm{~mW}$. The received optical power was $49.6 \mathrm{~mW}$. These powers were measured by an optical power meter (Thorlabs, S121C). Details of the circuit model for simultaneous energy harvesting and data transmission can be found in the Supplementary Information. The signal was captured by an oscilloscope (Keysight, MSO7104B) and sent to the laptop to be processed in MATLAB. The SNR was measured based on a training sequence, and for comparison, it was also theoretically approximated based on the estimated optical channel gain, noise power, and OPV parameters (capacitance, shunt resistance, etc.). Adaptive bit and power loading, as explained in the previous section, was used to determine the maximum modulation depth at each subcarrier. A sequence of random data bits was transmitted, and the BER was measured. The power dissipated by the load resistor in the energyharvesting branch was regarded as the harvested power, which could be obtained from the voltage across the resistor and the current flowing through it.

\section{Acknowledgements \\ H.H. acknowledges the financial support from the Wolfson Foundation and Royal Society. He also acknowledges financial support from the Engineering and Physical Sciences Research Council (EPSRC) under the Established Career Fellowship grant EP/R007101/1. The authors acknowledge the EPSRC for financial support from the program/project grants EP/K00042X/1 and EP/ R005281/1. L.K.J. acknowledges support from a Marie Skłodowska-Curie Individual Fellowship (European Commission) (MCIF: no. 745776)}

\section{Author details}

${ }^{1}$ LiFi Research and Development Centre, Department of Electronic \& Electrical Engineering, The University of Strathclyde, Technology \& Innovation Centre, 99 
George Street, Glasgow G1 1RD, UK. ${ }^{2}$ Organic Semiconductor Centre, SUPA, School of Physics and Astronomy, University of St Andrews, St Andrews KY16 9SS, UK. ${ }^{3}$ pureLiFi, Rosebery House, 9 Haymarket Terrace, Edinburgh EH12 5EZ, UK

\section{Author contributions}

H.H., I.D.W.S., and G.A.T. conceived of the project and supervised the research. S.V., P.P.M., and I.T. planned the project, guided by H.H., I.D.W.S., and G.A.T. L.K.J. designed and fabricated OPVs under the supervision of I.D.W.S. P.P.M. built the apparatus for measuring the frequency response of the OPVs and performed the measurements with L.K.J. I.T. and R.B. designed the SISO and MIMO communication experiments, assessed the system performance (i.e., BER, data rate, etc.) and derived the MIMO communication channel capacity. I.T., R.B. L.K.J., P.P.M., H.H., I.D.W.S., and G.A.T. wrote the manuscript.

\section{Data availability}

The authors declare that all data supporting the findings of this study are available within the paper and its supplementary information files. The source data underlying Figs. 3, 4, 6, and 7b of the main paper and supplementary Figs. S1-S4 and S6-S8 are provided as source data files at https://doi.org/10.15129/ 01 bf34cf-889a-4f8c-b1ad-8bf482443228.

\section{Conflict of interest}

The authors declare no competing interests.

Supplementary information The online version contains supplementary material available at https://doi.org/10.1038/s41377-021-00487-9.

Received: 24 June 2020 Revised: 9 January 2021 Accepted: 2 February 2021 Published online: 23 February 2021

\section{References}

1. Winzer, P. J. \& Neilson, D. T. From scaling disparities to integrated parallelism: a decathlon for a decade. J. Lightwave Technol. 35 1099-1115 (2017).

2. Cisco edge-to-enterprise loT analytics for electric utilities solution overview. https:/mww.cisco.com/c/dam/global/fr_fr/solutions/data-centervirtualization/big-data/solution-cisco-sas-edge-to-entreprise-iot.pdf (2018).

3. Cogalan, T. \& Haas, H. Why would $5 \mathrm{G}$ need optical wireless communications? Proceedings of the IEEE 28th Annual International Symposium on Personal, Indoor, and Mobile Radio Communications (PIMRC). 1-6 (IEEE, 2017).

4. Haas, H., Elmirghani, J. \& White, I. Optical wireless communication. Philos. Trans. R. Soc. A: Math. Phys. Eng. Sci. 378, 20200051 (2020).

5. Haas, H. et al. What is LiFi? J. Lightwave Technol. 34, 1533-1544 (2015).

6. Islim, M. S. et al. Towards $10 \mathrm{~Gb} / \mathrm{s}$ orthogonal frequency division multiplexingbased visible light communication using a GaN violet micro-LED. Photon Res. 5, A35-A43 (2017).

7. Fakidis, J. et al. 0.5-Gb/s OFDM-based laser data and power transfer using a GaAs photovoltaic cell. IEEE Photon. Technol. Lett. 30, 841-844 (2018).

8. Zhang, X. B. et al. Wide-coverage beam-steered 40-Gbit/s non-line-of-sight optical wireless connectivity for Industry 4.0. J. Lightwave Technol. 38, 6801-6806 (2020).

9. Lian, J. et al. Indoor visible light communications, networking, and applications. J. Phys. Photonics 1, 012001 (2019).

10. Khalighi, M. A., Akhouayri, H. \& Hranilovic, S. Silicon-photomultiplier-based underwater wireless optical communication using pulse-amplitude modulation. IEEE J. Ocean. Eng. 45, 1611-1621 (2019).

11. Miramirkhani, F. \& Uysal, M. Channel modelling for indoor visible light communications. Philos. Trans. R. Soc. A: Math. Phys. Eng. Sci. 378, 20190187 (2020).

12. Bian, R., Tavakkolnia, I. \& Haas, H. $15.73 \mathrm{~Gb} / \mathrm{s}$ visible light communication with off-the-shelf LEDs. J. Lightwave Technol. 37, 2418-2424 (2019).

13. Wang, Z. X. et al. Towards self-powered solar panel receiver for optical wireless communication. Proceedings of 2014 IEEE International Conference on Communications (ICC), 3348-3353 (IEEE, 2014).

14. Wang, Z. X. et al. On the design of a solar-panel receiver for optical wireless communications with simultaneous energy harvesting. IEEE J. Sel. Areas Commun. 33, 1612-1623 (2015).
15. Lorrière, N. et al. Photovoltaic solar cells for outdoor LiFi communications. J. Lightwave Technol. 38, 3822-3831 (2020).

16. Rühle, K., Glunz, S. W. \& Kasemann, M. Towards new design rules for indoor photovoltaic cells. Proceedings of the 38th IEEE Photovoltaic Specialists Conference, 2588-2591 (IEEE, 2012).

17. Cutting, C. L., Bag, M. \& Venkataraman, D. Indoor light recycling: a new home for organic photovoltaics. J. Mater. Chem. C. 4, 10367-10370 (2016).

18. Lee, H. K. H. et al. Organic photovoltaic cells-promising indoor light harvesters for self-sustainable electronics. J. Mater. Chem. A 6, 5618-5626 (2018).

19. Pacchioni, G. Organic electronics: packing tips for charge transport. Nat. Rev Materials 1, 16005 (2016)

20. Chiechi, R. C. et al. Modern plastic solar cells: materials, mechanisms and modeling. Mater. Today 16, 281-289 (2013).

21. Li, G., Zhu, R. \& Yang, Y. Polymer solar cells. Nat. Photonics 6, 153-161 (2012).

22. Chen, X. B., Min, C. Y. \& Guo, J. Q. Visible light communication system using silicon photocell for energy gathering and data receiving. Int. J. Opt. 2017, 6207123 (2017).

23. Sarwar, R. et al. Visible light communication using a solar-panel receiver. Proceedings of the 16th International Conference on Optical Communications and Networks (ICOCN), 1-3 (IEEE, 2011).

24. Wu, J. T. et al. Performance enhancement technique of visible light communications using passive photovoltaic cell. Opt. Commun. 392, 119-122 (2017).

25. Shin, W. H. et al. Self-reverse-biased solar panel optical receiver for simultaneous visible light communication and energy harvesting. Opt. Express 24, A1300-A1305 (2016).

26. Zhang, S. Y. et al. Organic solar cells as high-speed data detectors for visible light communication. Optica 2, 607-610 (2015).

27. Arredondo, B. et al. Visible light communication system using an organic bulk heterojunction photodetector. Sensors 13, 12266-12276 (2013).

28. Jørgensen, M., Norrman, K. \& Krebs, F. C. Stability/degradation of polymer solar cells. Sol. Energy Mater. Sol. Cells 92, 686-714 (2008).

29. Wang, K. et al. Inverted organic photovoltaic cells. Chem. Soc. Rev. 45 2937-2975 (2016).

30. Rajbhandari, S. et al. A multigigabit per second integrated multiple-input multiple-output VLC demonstrator. J. Lightwave Technol. 35, 4358-4365 (2017).

31. Fath, T. \& Haas, H. Performance comparison of MIMO techniques for optical wireless communications in indoor environments. IEEE Trans. Commun. 61, 733-742 (2013).

32. Yan, C. Q. et al. Non-fullerene acceptors for organic solar cells. Nat. Rev. Mater. 3, 18003 (2018).

33. Zhang, J. Q. et al. Material insights and challenges for non-fullerene organic solar cells based on small molecular acceptors. Nat. Energy 3, 720-731 (2018).

34. Jagadamma, L. K. et al. Polymer solar cells with efficiency $>10 \%$ enabled via a facile solution-processed Al-doped $\mathrm{ZnO}$ electron transporting layer. Adv. Mater. Mater. 5, 1500204 (2015).

35. Holliday, S. et al. High-efficiency and air-stable P3HT-based polymer solar cells with a new non-fullerene acceptor. Nature. Nat. Commun. 7, 11585 (2016).

36. Hou, J. H. et al. Organic solar cells based on non-fullerene acceptors. Nat. Mater. 17, 119-128 (2018).

37. Li, Y. X. et al. Non-fullerene acceptor with low energy loss and high external quantum efficiency: towards high performance polymer solar cells. J. Mater. Chem. A 4, 5890-5897 (2016).

38. Zhang, G. Y. et al. Nonfullerene acceptor molecules for bulk heterojunction organic solar cells. Chem. Rev. 118, 3447-3507 (2018).

39. Weinstein, S. B. The history of orthogonal frequency-division multiplexing [History of Communications]. IEEE Commun. Mag. 47, 26-35 (2009).

40. Tsonev, D., Videv, S. \& Haas, H. Unlocking spectral efficiency in intensity modulation and direct detection systems. IEEE J. Sel. Areas Commun. 33, 1758-1770 (2015).

41. Narmanlioglu, O. et al. Link adaptation for MIMO OFDM visible light communication systems. IEEE Access 5, 26006-26014 (2017).

42. Levin, H. E. A complete and optimal data allocation method for practical discrete multitone systems. Proceedings of 2001 IEEE Global Telecommunications Conference (GLOBECOM), 369-374 (IEEE, 2001).

43. Zhang, L. M. \& Kschischang, F. R. Staircase codes with $6 \%$ to $33 \%$ overhead. J. Lightwave Technol. 32, 1999-2002 (2014).

44. Jagadamma, L. K. et al. Efficient inverted bulk-heterojunction solar cells from low-temperature processing of amorphous ZnO buffer layers. J. Mater. Chem. A 2, 13321-13331 (2014). 